Article

\title{
Multiscale Modeling of the Early CD8 T-Cell Immune Response in Lymph Nodes: An Integrative Study
}

\author{
Sotiris A.Prokopiou $^{1, *}$, Loic Barbarroux ${ }^{1,2}$, Samuel Bernard ${ }^{1,2}$, Julien Mafille ${ }^{3,4,5,6}$, \\ Yann Leverrier $^{3,4,5,6}$, Christophe Arpin ${ }^{3,4,5,6}$, Jacqueline Marvel ${ }^{3,4,5,6}$, Olivier Gandrillon ${ }^{1,7}$ \\ and Fabien Crauste ${ }^{1,2}$ \\ ${ }^{1}$ Inria team Dracula, Inria center Grenoble Rhone-Alpes, 66 Boulevard Niels Bohr F-69603 \\ Villeurbanne, France; E-Mails: loic.barbarroux @inria.fr (L.B.); samuel.bernard@inria.fr (S.B.); \\ olivier.gandrillon@univ-lyon1.fr (O.G.); fabien.crauste@inria.fr (F.C.) \\ ${ }^{2}$ Université Lyon 1, CNRS UMR 5208, Institut Camille Jordan, 43 blvd du 11 novembre 1918, \\ F-69622 Villeurbanne-Cedex, France \\ ${ }^{3}$ Inserm, U1111, Lyon F-69007, France; E-Mail: julien.mafille@inserm.fr (J.Maf.); \\ yann.leverrier@inserm.fr (Y.L.); christophe.arpin@inserm.fr (C.A.); \\ jacqueline.marvel@inserm.fr (J.Mar.) \\ ${ }^{4}$ CNRS, UMR5308, Lyon F-69007, France \\ ${ }^{5}$ Université Lyon 1, Centre International de Recherche en Infectiologie, Lyon F-69007, France \\ ${ }^{6}$ Ecole Normale Supérieure de Lyon, Lyon F-69007, France \\ ${ }^{7}$ Université Lyon 1, Centre de Génétique et de Physiologie Moléculaire et Cellulaire, \\ F-69622 Villeurbanne-Cedex, France \\ * Author to whom correspondence should be addressed; E-Mail: sotirisprokopiou1 @ gmail.com; \\ Tel.: +1-813-745-7351; Fax: +1-813-745-6497.
}

External Editor: Filippo Castiglione

Received: 28 May 2014; in revised form: 18 July 2014 / Accepted: 4 September 2014 /

Published: 29 September 2014

\begin{abstract}
CD8 T-cells are critical in controlling infection by intracellular pathogens. Upon encountering antigen presenting cells, T-cell receptor activation promotes the differentiation of naïve CD8 T-cells into strongly proliferating activated and effector stages. We propose a 2D-multiscale computational model to study the maturation of CD8 T-cells in a lymph node controlled by their molecular profile. A novel molecular pathway is presented and converted into an ordinary differential equation model, coupled with a cellular Potts model to describe cell-cell interactions. Key molecular players such as activated IL2 receptor and Tbet levels
\end{abstract}


control the differentiation from naïve into activated and effector stages, respectively, while caspases and Fas-Fas ligand interactions control cell apoptosis. Coupling this molecular model to the cellular scale successfully reproduces qualitatively the evolution of total CD8 T-cell counts observed in mice lymph node, between Day 3 and 5.5 post-infection. Furthermore, this model allows us to make testable predictions of the evolution of the different CD8 T-cell stages.

Keywords: multiscale immune modeling; cellular Potts model; CD8 T-cells; APC

\section{Introduction}

During viral infection in a tissue, resident antigen presenting cells (APC), such as dendritic cells (DCs), uptake antigen and recirculate via lymphatics to reach a nearby lymph node (LN) within 2-3 days [1], where they encounter different types of immune cells (e.g., B- and T-cells) including CD8 T-cells. The establishment of cellular contact between a CD8 T-cell and an APC provides the opportunity for antigen recognition to occur through CD8 T-cell receptor (TCR) interactions with peptide-major histocompatibility complexes (MHC) that are present at the surface of the APC (activation phase). In addition, CD8 T-cell-APC contacts involve various other receptor-ligand interactions (including those of co-stimulatory and adhesion molecules [2]) and the delivery of soluble mediators (e.g., interleukin 2, IL2) that modulate the outcome of CD8 T-cell activation. Productive contacts (in terms of timing and strength) between naïve CD8 T-cells and APC result in the differentiation of naïve CD8 T-cells into activated, effector and memory CD8 T-cell stages and, thereby, in their clonal expansion. In particular, the short-lived cytotoxic effector cells elaborate cytokines and granzymes to kill pathogen-infected cells and control the infection [3]. Following pathogen clearance, most effector T-cells undergo cell apoptosis (contraction phase), while 5\%-10\% of them survive and mature into long-lived memory CD8 T-cells [4]. CD8 T-cells specific for a given peptide only consist of a small fraction of naïve repertoire; therefore, robust proliferation is required to combat rapidly replicating pathogens [5].

In LNs, CD8 T-cells receive signals during both short-lived contacts and long-lived interactions with APC. These contacts are highly regulated events and are influenced by the timing of activation via the TCR, the signal strength (antigen load together with the binding affinity of TCR to MHC I-peptide complex), the inflammatory environment (e.g., interferon-gamma and interleukin-12) and the presence of other responding T-cells. For these interactions to occur, cell motility plays a crucial role. The rate of CD8 T-cell-APC contacts is not only the result of random collision events [6]. Chemokines produced at the site of CD8 T-cell-APC conjugates [2] and the extracellular environment (e.g., fibroblastic reticular cell network) might bias the direction of cell movement [6,7].

An activated CD8 T-cell is exposed to a myriad of signals and environmental cues. How these signals are transmitted into cells and translated into gene expression patterns that promote T-cell proliferation and differentiation is an important and complex question [8]. 
Mathematical models of the immune response in the lymph nodes may provide important insights into how interactions between CD8 T-cells and APC can lead to specific results. Different types of mathematical models have been used to model different aspects of the immune response, including continuous models [9,10]. These models describe cell populations as densities, which makes the consideration of the role of CD8 T-cell and APC properties, such as cell shape and cell adhesion (important for modeling cell-cell interactions that lead to T-cell activation), mathematically complicated. Therefore, individual-based models (IBMs), where each cell is treated as an individual entity, are more appropriate.

Beltman et al. [11] used a particular type of IBM, the Glazier-Graner-Hogeweg model (also called the Cellular Potts Model (CPM) [12]), to model the interactions between T-cells and DCs in the LN paracortex. The 3D space is filled with static rods, representing the reticular network. T-cells randomly move in the domain, and their movements are not biased by any external factors (for instance, chemotaxis or haptotaxis). DCs, however, are less motile but they extend their dendrites (long projections) to facilitate contact with T-cells. When T-cells and DCs come into contact, T-cells decrease their motility because of an increase of the strength of adhesion and putative "stop signals" that antigen-bearing DCs transmit to T-cells. The authors investigated which circumstances are required to obtain long-lived interactions between T-cells and DCs, and they focused on how the migratory behavior of T-cells is affected by changes in their adhesion properties and in the 'stop signals' transmitted by DCs, as well as by the dynamic behavior of DC's dendrites. Simulations suggested that the initial decrease in T-cell motility after antigen appearance is mainly due to "stop signals" transmitted by activated DCs to T-cells.

In addition, Gong et al. [13] used a 3D IBM (where cells were modeled as non-deformable spheres), which allows for differentiation into effector cells in different antigenic conditions in the LN. The authors suggested that a 3D model (compared to 2D) is more appropriate to analyze LN function, since T-cell encounters DCs and TCR repertoire scanning is more efficient. The results suggested that reducing the time naïve T-cells need to bind DCs before becoming activated would increase the rate at which effector cells are produced.

Riggs et al. [14] used a 2D IBM to compare chemotaxis with random T-cell motion in the LN and showed that chemotaxis increased the total number of T-cell-DC contacts, but decreased the number of contacts with a given T-cell, ultimately producing fewer activated T-cells. Results suggested that, within an LN T-zone (the subcortical zone where T-cells mainly interact with DCs), a random search strategy is optimal for a rare cognate T-cell to find its DC match and maximize the production of activated T-cells.

LNs may become larger during infection, due to a reduced ability of T-cells to exit the LN, an increase in the T-cell flow to the $\mathrm{LN}$ and the T-cell proliferation within those LN [15], an important phenomenon with implications for T-cell-APC interactions, which has been initially addressed by Bogle and Dunbar [16]. In particular, the latter authors allowed their 3D simulation domain to grow during an immune response, although they did not study in detail how this expanding LN affects output.

Baltazzi et al. [17] used a 3D hybrid discrete/continuous model of the LN focusing on the lymphocyte recruitment and trafficking (accounting for the effect of chemotaxis on cell motility) inside the lymph node. The model tracks the evolution of three types of cells: Th (helper) cells, B-cells and DCs. Each type of cell consists of different (e.g., antigen, chemokine) receptors (e.g., CXCR5, CCR7, S1P1), where each one is randomly chosen to be expressed or not, affecting the status of each cell. The 
model reproduces first the expected dynamics of the system with and without the presence of antigenic stimulation and also investigates the role of DCs and S1P1-control of lymphocyte exit from LN. It is shown that DC removal at early times produces large effects, with an immune response that is greatly delayed and reduced in magnitude due to the lack of active Th cells that are able to provide the right co-stimulation to B-lymphocytes. S1P1 control, instead, affects essentially the magnitude of the immune response rather than the timing, by decreasing the overall number of specific lymphocytes that can participate in antigen detection.

One of the complexities of the immune response lies within its intrinsic multiscale nature. All of the previous cited studies described cell-cell interactions without an explicit description of the molecular events involved. On the other hand, a number of studies investigated the functioning of the molecular responses [18,19] without an explicit description of the cell-cell interaction level. In this work, we aimed at providing the first multiscale model of the early immune CD8 T-cell response within an LN, based on the description of the first observable activation stages of CD8 T-cells; that is, naïve, preactivated, activated, and effector stages. An explicit description of a molecular regulatory network (based upon an ordinary differential equation (ODE) system) is coupled with the cellular and extracellular levels (based upon the use of a CPM and a partial differential equation (PDE), respectively) in order to observe the emergence of the global tissue behavior. Our model successfully recapitulates qualitative features of the experimental evolution of the total CD8 T-cells in draining LN upon infection, and can make predictions about the evolution of the different CD8 T-cell differentiation stages.

Figure 1. Experimental CD8 T-cell counts over time in mice lymph nodes. The data were collected following intranasal H1N1 infection in C57B1/6 mice (adoptively transferred with $\mathrm{F} 5$ cells $24 \mathrm{~h}$ prior to the infection). The data used were from two independent experiments with different kinetics and two to four mice per time point. Error bars show the SEM and the average is marked in blue ("x").

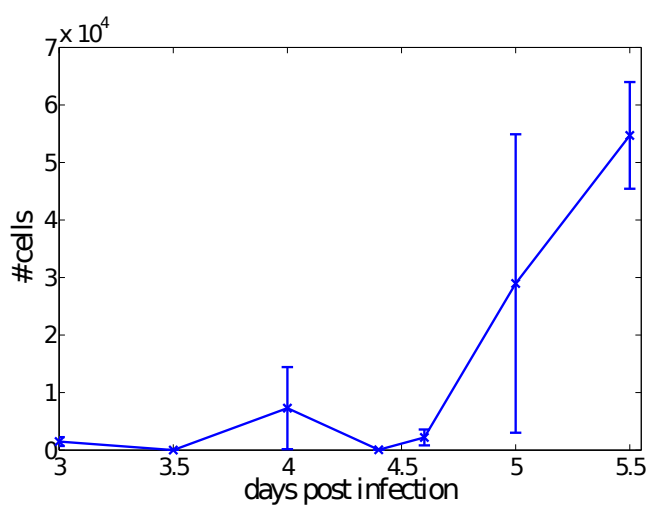

\section{Experimental Section}

Two hundred thousand CD8 T-cells from CD45.1+ F5 TCR transgenic mice (B6.SJL-Ptprc ${ }^{a}$ Pepc $^{b} /$ BoyCrl-Tg(CD2-TcraF5,CD2-TcrbF5)1Kio/Jmar) recognizing the NP68 epitope were transferred by retro-orbital injection in congenic CD45.2+ C57B1/6 mice (C57BL6/J). The data used were from two independent experiments with different kinetics and two to four mice per time point. The day after mice were inoculated intranasally with $2.10^{5} \mathrm{TCID}_{50}$ of an H1N1 Influenza 
virus expressing the NP68 epitope. From Day 3 to 5.5 post-infection (Figure 1), the lymph node draining the site of infection (lung, mediastinal lymph node) was harvested at different time points, and the number of F5 transgenic CD8 responder T-cells was assessed by flow cytometry, based on CD8/CD45.1/CD45.2 expression. All experimental procedures were approved by our local animal experimentation ethics committee (CECCAPP; Lyon, France), and accreditations have been obtained from French governmental agencies.

\section{Multiscale Model}

In this section, we describe the three different levels of our cell-based, multiscale model. One should note that we aim in this work at describing early LN events (see Figure 1) during a time frame where no memory cells yet appear. We therefore did not include into our model the molecular events leading to the generation of memory cells. Differentiation only proceeds up to the effector stage from the naïve and through a preactivated and activated stage.

\subsection{Subcellular Level}

In the following, we describe a novel molecular regulatory pathway of T-cell activation (Figure 2). This is a simplified model which, although not describing the full picture, provides all of the necessary information for the coupling between the intracellular molecular level and cellular fates (differentiation and apoptosis). The dynamical state of the molecular model will decide cell proliferation (TCR activation and IL2 production), cell apoptosis (activation of caspases) and cell differentiation (via the level of expression of the activated IL2 receptor and the protein, Tbet).

Figure 2. Simplified molecular signaling pathway of a CD8 T-cell. The molecular network gets activated by T-cell receptor (TCR) signaling once a T-cell encounters an antigen presenting cell (APC). The activated IL2 receptor (IL2R*) plays an essential role in the differentiation of a naïve T-cell into its activated stage, and Tbet into its effector stage. All numbers refer to Table 1. Solid curves, positive feedback; dashed curves, negative feedback.

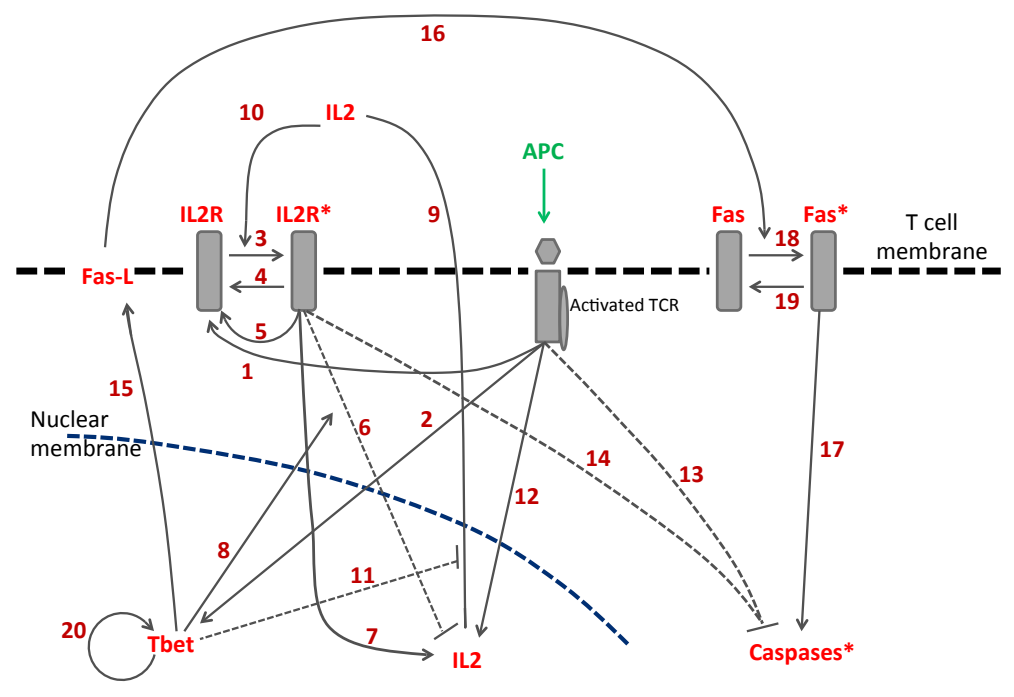


Table 1. Description of the molecular signaling pathways in Figure 2.

\begin{tabular}{lll}
\hline & Description & Reference \\
\hline 1. & Activated TCR increases the amount of non-activated IL2R & {$[20]$} \\
2. & Activated TCR activates Tbet & {$[21]$} \\
3. & Transition: from low to activated IL2R & {$[19]$} \\
4. & Transition: from high to non-activated IL2R & {$[19]$} \\
5. & Activated IL2R increases the amount of non-activated IL2R & {$[20]$} \\
6. & Activated IL2R inhibits the expression of IL2 gene (via Blimp1) & {$[22]$} \\
7. & Activated IL2R induces the expression of $I L 2$ gene & {$[20]$} \\
8. & Tbet promotes the inhibition of IL2 gene expression (via Blimp1) & {$[23]$} \\
9. & Internal IL2 gets secreted and gives rise to the external IL2 & {$[19]$} \\
10. & External IL2 enhances the transition from non-activated to activated IL2R & {$[20]$} \\
11. & Tbet inhibits the secretion of IL2 & {$[24,25]$} \\
12. & IL2 gene activation from TCR (via Erk) & {$[20]$} \\
13. & TCR inhibits caspase (via Erk and Bim/Bax/Bcl2) & {$[26]$} \\
14. & Activated IL2R inhibits caspase (via Stat5, Bcl2, and BAX) \\
15. & Tbet induces the expression of FasL & {$[27]$} \\
16. & FasL activates Fas through cell contact & {$[28]$} \\
17. & activated Fas (Fas*) induces caspase activation & {$[29]$} \\
18. & Transition: from non-activated to activated form of Fas \\
19. & Transition: from activated to non-activated form of Fas \\
20. & Tbet maintenance & {$[30,31]$} \\
\hline & & {$[29]$} \\
\hline
\end{tabular}

CD8 T-cell proliferation follows TCR stimulation and production of IL2 (Figure 2). TCR stimulation induces the upregulation of IL2 gene expression [20] and expression of the non-activated IL2 receptor (aggregating all steps toward the formation of the active form of the receptor), IL2R [20]. Following IL2 secretion by the CD8 T-cell, IL2 binds to IL2R [20] to form the activated IL2 receptor (that is the quaternary ligand/receptor complex IL2Ra (CD25)/IL2Rb (CD122)/IL2Rg (CD132)/IL2), IL2R* [19]. IL2 $\mathrm{R}^{*}$ can favor the production of IL2R and the upregulation of the IL2 gene [20], but also down-regulates the IL2 gene expression (through Blimp1) [22], enhanced by Tbet (encoded by Tbx21), a T-box transcription factor [23].

Caspases, on the other hand, play essential roles in cell apoptosis. Although there are several types of caspases, we do not make any distinction among them and use caspases in a more generic form. The activation of T-cells leads to the expression of the surface protein Fas-ligand (denoted by FasL), 
the ligand of protein Fas, via the activation of Tbet protein [28]. Fas-induced apoptosis is one of the main mechanisms by which cytotoxic T-lymphocytes induce cell death in cells expressing foreign antigens [32]. Perforin and Granzyme B also play a role in inducing cell death [28], yet we do not explicitly describe their role. Fas-FasL signaling activated by cell contact has a positive impact on caspases [29], which is inhibited by both TCR activation and the IL2R* (through Stat5 in the IL2R signaling cascade) [26,27].

Although the precise processes involved in the generation of activated and effector stages remain undefined, IL2R* and Tbet have been found to play an important role in activated and effector cell fate decisions, respectively [3,33]. Stimulated TCR activates Tbet [21], and the increment of Tbet levels might be an effect of both direct autoregulatory regulation [30] and other intermediate pathways (e.g., EGR1 [31]), even though evidence has only been obtained for CD4 T-cells [30,31]. Arrow 20 in Figure 2 represents both the Tbet self-maintenance and also the contribution from other molecular elements. Tbet was also demonstrated to suppress IL2 secretion [24,25].

The novelty in the current study is the coupling between the cellular level (differentiation of T-cells) and the molecular pathway as shown in Figure 3. The state of the dynamical molecular network described above and in Figure 2 defines the different CD8 T-cell stages, as follows: naïve (no prior contact with APC; no TCR activation), preactivated (came in contact with APC and its activated IL2R is below a threshold), activated (came in contact with APC and its activated IL2R is above a threshold) and effector (came in contact with APC and Tbet is above a threshold).

Figure 3. Schematic diagram of the linear activation pathway for the CD8 T-cells. Due to TCR signaling (as shown in Figure 2), once a naïve T-cell comes into contact with an APC it becomes preactivated, then evolves to an activated cell when its IL $2 \mathrm{R}^{*}$ dynamically crosses a threshold and, eventually, to an effector stage when Tbet dynamically crosses a threshold. The caspase activation level is essential for cell apoptosis. Fas-FasL signaling further enhances apoptosis of activated and effector stages if activated-effector or effector-effector cells come into contact (cytotoxic effect). In contrast, the death of APC is independent of caspase levels, because the molecular network does not apply for APC. Key: N, naïve; PA, preactivated; A, activated; E, effector; APC, antigen presenting cell.

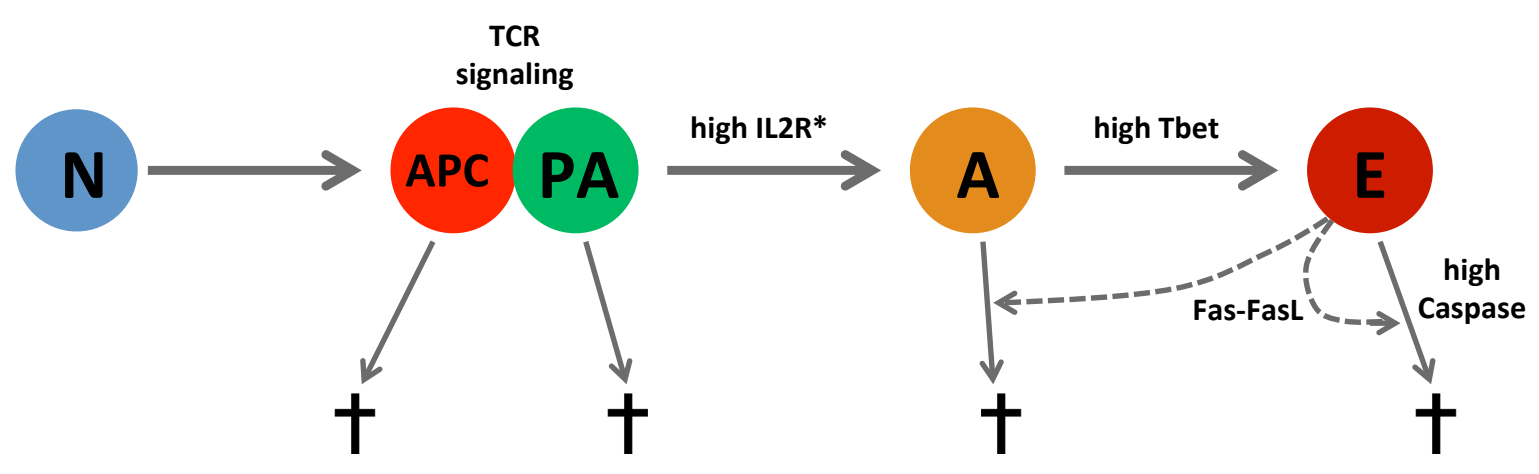


The complex dynamics of the molecular pathway presented in Figure 2 can be converted in the following nonlinear ODE model:

$$
\begin{aligned}
& \text { Non-activated IL2R: } \frac{d[I R]}{d t}=\lambda_{R 1} f(A P C)+\left(\lambda_{R 2}+\mu_{I L 2}^{-}\right)\left[I R^{*}\right]-\mu_{I L 2}^{+}\left[I L 2^{c m}\right][I R]-k_{R}[I R], \\
& \text { Activated IL2R: } \frac{d\left[I R^{*}\right]}{d t}=\mu_{I L 2}^{+}\left[I L 2^{c m}\right][I R]-\mu_{I L 2}^{-}\left[I R^{*}\right]-k_{R^{*}}\left[I R^{*}\right], \\
& \text { Tbet: } \frac{d[T b]}{d t}=\lambda_{T 1} f(A P C)+\lambda_{T 2} \frac{[T b]}{\lambda_{T 3}+[T b]}[T b]-k_{T}[T b], \\
& \text { Non-activated Fas: } \frac{d[F s]}{d t}=\lambda_{F}+\mu_{F}^{-}\left[F s^{*}\right]-H \mu_{F}^{+}\left[T b^{c m}\right][F s]-k_{F}[F s], \\
& \text { activated Fas: } \frac{d\left[F s^{*}\right]}{d t}=H \mu_{F}^{+}\left[T b^{c m}\right][F s]-\mu_{F}^{-}\left[F s^{*}\right]-k_{F^{*}}\left[F s^{*}\right], \\
& \text { Caspase: } \quad \frac{1[C]}{d t}=\lambda_{c 1}\left(\frac{1}{1+\lambda_{c 2}\left[I R^{*}\right]}\right) \cdot\left(\frac{1}{1+\lambda_{c 3} f(A P C)}\right)+\lambda_{c 4}\left[F s^{*}\right]-k_{C}[C],
\end{aligned}
$$

with most of the parameters $\lambda$ representing the strengths of feedbacks (except for $\lambda_{F}$ : the baseline rate of transcription of Fas in naïve T-cells). Parameters $k$ with a subscript $R, R^{*}, T, F, F^{*}, C$ are the decay rates of corresponding variables, and $\mu^{+}, \mu^{-}$with subscripts $I L 2, F$ are the association and dissociation rates for IL2R and Fas, respectively.

T-cells appear to sum up TCR signals that are delivered during early and late encounters with APCs, which is a feature that helps them sense the overall amount of antigen available in the LN [2]. As a consequence, the quality and the functional heterogeneity of a T-cell response might be shaped by the history of contacts that have been experienced by individual T-cells. Therefore, in Equations (1), (3) and (6) we chose $f(A P C)$ to represent the strength of TCR signaling and, more specifically, to be the number of simultaneous contacts of a T-cell with APCs. Therefore, if a T-cell is not in contact with any APC, $f(A P C)=0$, which implies no TCR activation, and if, for instance, a T-cell is in contact with two APCs, then $f(A P C)=2$. In reality, only a small fraction of T-cells will express T-cell receptors specific for a given epitope. Yet, in our model we do not make this distinction since our experimental data have been generated using F5 transgenic cells, for which naïve cells all express the TCR recognizing the NP68 antigen.

The single-cell ODE dynamical system (1)-(6) is coupled to neighbor cells via two terms, $I L 2^{\mathrm{cm}}$ and $T b^{c m}$. In Equations (1) and (2), IL2 ${ }^{\mathrm{cm}}$ represents the concentration of IL2 at the boundary (cell membrane) of each T-cell. In Equations (4) and (5), $T b^{c m}$ is the sum of all Tbet concentrations from all of the neighbor cells in contact. In fact, $T b^{c m}$ implicitly describes the FasL-induced caspase activation, since we decided not to explicitly describe the Fas-FasL interaction for the sake of simplicity.

In Equations (4) and (5), $H$ is the Heaviside function where $H=1$ if an effector cell comes into contact with at least another effector or activated cell, and $H=0$, otherwise. It represents the Fas-FasL interaction which promotes the upregulation of caspases. 


\subsection{Cellular Level: Cellular Potts Model}

In the lattice-based CPM, cells are represented as spatially-extended, discrete objects with explicit cell shapes evolving in a 2D computational domain. The cell interactions are characterized through a total energy:

$$
E=\sum_{\left(\vec{x}, \overrightarrow{x^{\prime}}\right)} J_{\tau(\sigma(\vec{x})), \tau\left(\sigma\left(\overrightarrow{x^{\prime}}\right)\right)}\left(1-\delta_{\sigma(\vec{x}), \sigma\left(\overrightarrow{x^{\prime}}\right)}\right)+\lambda_{\text {area }} \sum_{\sigma>0}\left(a_{\sigma}-A_{\sigma}\right)^{2}+E^{\prime}
$$

where $J$ are the contact energies between cells, $a_{\sigma}$ the cell area and $A_{\sigma}$ the target cell area. In addition, $\tau(\sigma)$ represents the type of the cell occupying a grid space $\sigma$, which in our model can be either an APC or one of the four T cell stages (naïve; preactivated; activated; effector). The Kronecker delta function is $\delta_{x, y}=1$, if $x=y$; 0 , if $x \neq y$, and the term $\left(1-\delta_{\sigma(\vec{x}), \sigma\left(\overrightarrow{x^{\prime}}\right)}\right)$ ensures that adhesive energy only accrues at cell surfaces (not inside the cells); and $E^{\prime}$ can be any constraint on the cell behavior (e.g., chemotaxis). To mimic cytoskeletally-driven, active surface fluctuations, a lattice site $\vec{x}$ and a neighboring target $\overrightarrow{x^{\prime}}$ are randomly selected. Then, we calculate how the effective energy would change if the initial site displaced the target. If the energy decreases $\left(\Delta E=E_{\text {new }}-E_{\text {old }}<0\right)$, the change occurs with probability one. However, if $E$ increases $(\Delta E>0)$, the change will be accepted with Boltzmann probability, $p=\exp (-\Delta E / T)$, where $T$ is the 'temperature' of the system; $T$ influences the likelihood of energetically unfavorable events taking place: the higher $T$, the more out-of-equilibrium the system will be. Biologically, the $T$ indicates the amplitude of cell membrane fluctuations (and not active cell movement), which is characterized by polarized membrane extension and retraction. On a lattice with $M$ sites, $M$ site copy attempts represent our basic unit of time, one Monte Carlo Step (MCS).

For our computational results presented in the next section, we use the open-source simulation environment CompuCell3D (CC3D) [34]. The source code is freely available upon request to the first author. Our model is formulated on a square domain (size $200 \mu \mathrm{m} \times 200 \mu \mathrm{m}$ ).

By equating the initial cell volume in the model to the real cell size (e.g., $5 \times 5$ pixels $\left.=10 \times 10 \mu \mathrm{m}^{2}[35]\right)$, we can convert the lattice spacing to microns $\left(1 \mathrm{pixel}=4 \mu \mathrm{m}^{2}\right)$. In addition, we set the time conversion to be $1 \mathrm{MCS}=1 \mathrm{~min}$, a value similar to those used in other CPM studies [36].

In Table 2, we summarize the different behaviors of the APC and T cells, which are discussed in detail in the following text.

To allow for cell-cell interactions, we expand the widely used CPM to incorporate cell motility. This is achieved by including an extra term in the total energy,

$$
E_{\text {motility }}^{\prime}=r(\cos \theta(t), \sin \theta(t)) \cdot\left(\vec{x}_{C M}-{\overrightarrow{x^{\prime}}}_{C M}\right)
$$

with $r$ representing the cell speed from its center of mass $\left(\vec{x}_{C M}=\sum_{i} \vec{x}_{i} / a_{\sigma}\right)$, where $i$ denotes pixels belonging to a given cell, and $\theta(t) \in[0,2 \pi]$ the rotation angle which updates every $t_{\text {per }}$ (the persistence time in MCS). The $t_{p e r}(=90 \mathrm{~min})$ refers to the average duration of locomotion in one direction before a random change of direction.

We assume that (all types of) T-cells move randomly in the domain with constant speed $\sim 0.75 \mu \mathrm{m} / \mathrm{min}(r=150)$, and they stop moving $(r=0)$ once they come into contact with APC [2,37]. 
In contrast to T-cells, APCs move rather slowly, $\sim 0.1 \mu \mathrm{m} / \mathrm{min}(r=20)$. The limitations on the maximum speed of cells that can be reached in the Potts model mainly depending on the dissociation of cells (break up into small pieces or fragments) above a certain value of $r$ led to smaller cell speed than the range mentioned in [2]. This could be considered for further investigation in a subsequent study.

Table 2. Behaviors of the APC and T cell stages.

\begin{tabular}{lcccc}
\hline Cell type $\backslash$ Behavior & Random motility & Division & Apoptosis & IL2 secretion \\
\hline APC & $\checkmark$ & X & $\checkmark$ & X \\
naïve & $\checkmark$ & X & X & X \\
preactivated & $\checkmark$ & X & $\checkmark$ & $\checkmark$ \\
activated & $\checkmark$ & $\checkmark$ & $\checkmark$ & $\checkmark$ \\
effector & $\checkmark$ & $\checkmark$ & $\checkmark$ & $\checkmark$ \\
\hline
\end{tabular}

Regarding cell division, the activated and effector T-cells proliferate every $8 \mathrm{~h}$ (see Supplementary Information of [38]). The molecular content of the parental cell is partitioned stochastically between the two daughters cells [39]. To achieve this, one daughter cell inherits the $k_{i} \in(0.7,1.0)$ fraction of the parent cell (for each molecular element in system (1)-(6)), and the other daughter inherits $2-k_{i}$. This will result in heterogeneity in the possible fate of the two daughter cells, a well-described phenomenon [40-42]. Although the exact range of variation of the molecular context that each of the two daughter cells inherit from the parent cell is unknown and difficult to track experimentally, we chose $(0.7,1.0)$ in order to include non-negligible, yet moderate, stochasticity.

Regarding cell death, APCs have a lifespan of 32-40 h [43,44], and therefore, each APC is randomly assigned a lifespan in this range taken from a uniform distribution. The lifespan of naïve T-cells lies in a range of 165-365 days (see Supplementary Information of [38]), but since this time frame is far from the scope of our model (which spans between day 3 and 5.5 post-infection), we assume no death of naïve T-cells. Except for naïve T-cells, all of the other CD8 T-cells (preactivated, activated and effector) die based on their molecular profile (once their caspase level reaches its threshold; see Table 5).

\subsection{Extracellular Level}

We consider one extracellular cytokine, IL2, one of the main regulatory cytokines of T-cell fate after activation [19], whose concentration evolves according to the following PDE:

$$
\frac{\partial[I L 2]}{\partial t}=D \nabla^{2}[I L 2]+\left(\lambda_{R 3} \frac{I R^{*}}{\lambda_{R 4}+I R^{*}}+\lambda_{1} f(A P C)\right) \frac{1}{1+\lambda_{T 4} T b}-\delta[I L 2]
$$

where $[I L 2]$ is the IL2 concentration, which diffuses in the square domain (with no flux boundary conditions), $D$, the diffusion coefficient, and $\delta$ the decay rate. The second term on the right-hand side represents the secretion rate, which is, for the first time, to our knowledge, controlled by molecular entities (TCR activation and IL2R*; see Figure 1). We set $D=0.2$ pixels/MCS, since the diffusion length $(L)=\sqrt{D / \delta} \sim 1$ cell length [18], and from there we can calculate $\delta=0.008 \mathrm{~min}^{-1}$. Note that, 
although unrealistic, the use of the CPM implies that IL2 identically diffuses through cell bodies and the extracellular space.

In Section 6.1, we provide a discussion on how the parameter values used in our simulations for the subcellular, cellular and extracellular level were estimated, and these are summarized in Tables 3-5.

Table 3. Default parameter settings for simulations: cellular level (Potts model)

\begin{tabular}{|c|c|c|c|}
\hline Parameter & Description & Value & Reference \\
\hline \multicolumn{4}{|c|}{ Cell features: } \\
\hline $\mathrm{A}_{T}$ & $\mathrm{~T}$ cell target area & $100 \mu m^{2}$ & {$[35]$} \\
\hline $\mathrm{A}_{A P C}$ & APC target area & $1000 \mu m^{2}$ & {$[35]$} \\
\hline $\mathrm{T}$ & cell-membrane fluctuations & 10 & / \\
\hline$\lambda_{\text {area }}$ & resistance to changes in size/area & 10 & l \\
\hline \multicolumn{4}{|l|}{ Adhesion: } \\
\hline$J_{T, m}$ & $\mathrm{~T}$ cell-medium contact energy & 30 & l \\
\hline$J_{A P C, m}$ & APC-medium contact energy & 30 & l \\
\hline$J_{A P C, A P C}$ & APC-APC contact energy & 100 & / \\
\hline$J_{A P C, N}$ & APC-naïve cell contact energy & 50 & l \\
\hline$J_{A P C, P A}$ & APC-preactivated cell contact energy & 50 & / \\
\hline$J_{A P C, A}$ & APC-activated cell contact energy & 500 & l \\
\hline$J_{A P C, E}$ & APC-effector cell contact energy & 500 & l \\
\hline$J_{T, T}$ & $\mathrm{~T}$ cell- $\mathrm{T}$ cell contact energy & 100 & / \\
\hline
\end{tabular}

Table 4. Default parameter settings for simulations: subcellular level (Key: M, molars)

\begin{tabular}{llll}
\hline Parameter & Value & Units & Reference \\
\hline Decay rates: & & & \\
$k_{R}$ & 0.0029 & $\mathrm{~min}^{-1}$ & {$[45,46]$} \\
$k_{R^{*}}$ & 0.0029 & $\mathrm{~min}^{-1}$ & {$[45]$} \\
$k_{T}$ & 0.0035 & $\mathrm{~min}^{-1}$ & {$[46]$} \\
$k_{F}$ & 0.0047 & $\mathrm{~min}^{-1}$ & {$[47]$} \\
$k_{F^{*}}$ & 0.0047 & $\mathrm{~min}^{-1}$ & {$[47]$} \\
$k_{C}$ & 0.0038 & $\mathrm{~min}^{-1}$ & {$[47]$} \\
\hline
\end{tabular}


Table 4. Cont.

\begin{tabular}{llll}
\hline Parameter & Value & Units & Reference \\
\hline Strengths of feedbacks: & & & \\
$\lambda_{R 1}$ & 0.0158 & $\mathrm{M} \mathrm{min}^{-1}$ & Derived \\
$\lambda_{R 2}$ & 0.001 & $\mathrm{~min}^{-1}$ & Derived \\
$\lambda_{T 1}$ & 0.01 & $\mathrm{M} \mathrm{min}^{-1}$ & Derived \\
$\lambda_{T 2}$ & 0.004 & $\mathrm{~min}^{-1}$ & {$[46]$} \\
$\lambda_{T 3}$ & 0.01 & $\mathrm{M}$ & $/$ \\
$\lambda_{c 1}$ & 0.01 & $\mathrm{M} \mathrm{min}$ & $/$ \\
$\lambda_{c 2}$ & 100 & $\mathrm{M}^{-1}$ & $/$ \\
$\lambda_{c 3}$ & 0.01 & $/$ & $/$ \\
$\lambda_{c 4}$ & 0.004 & $\mathrm{~min}^{-1}$ & $/$ \\
Association/Dissociation rates: & & & \\
$\mu_{I L 2}^{+}$ & $6 \times 10^{8}$ & $\mathrm{M}^{-1} \mathrm{~min}^{-1}$ & {$[46,48]$} \\
$\mu_{I L 2}^{-}$ & 0.006 & $\min ^{-1}$ & {$[19]$} \\
$\mu_{F}^{+}$ & $0.86 \times 10^{5}$ & $\mathrm{M}^{-1} \mathrm{~min}^{-1}$ & Derived \\
$\mu_{F}^{-}$ & 0.006 & $\min ^{-1}$ & {$[19]$} \\
Other: & & & \\
$\lambda_{F}$ & $3.47 \times 10^{-5}$ & $\mathrm{M} \mathrm{min}^{-1}$ & {$[46]$} \\
\hline
\end{tabular}

Table 5. Default parameter settings for simulations: extracellular level and molecular thresholds for T-cell differentiation and apoptosis.

\begin{tabular}{llll}
\hline Parameter & Value & Units & Reference \\
\hline Equation for IL2: & & & \\
$\lambda_{R 3}$ & 0.0 & $\mathrm{M} \mathrm{min}^{-1}$ & $/$ \\
$\lambda_{R 4}$ & 0.0 & $\mathrm{M}$ & $/$ \\
$\lambda_{T 4}$ & 0.0 & $\mathrm{M}^{-1}$ & $/$ \\
$\lambda_{1}$ & $10^{-12}$ & $\mathrm{M} \mathrm{min}^{-1}$ & {$[18]$} \\
Thresholds: & & & \\
IL2R $^{*}$ & 7 & $\mathrm{M}$ & Derived \\
Tbet & & $\mathrm{M}$ & Derived \\
Caspase $^{*}$ & 2.63 & $\mathrm{M}$ & Derived \\
\hline
\end{tabular}




\section{Results and Discussion}

In this section we present results from our in silico multiscale model. At first, we present results at the population level (cell counts), and in the next section we will explain the validation process at the intracellular level. We discuss the sensitivity of the model to some parameter values in Paragraph 4.3.

\subsection{Dynamics at the Population Level}

Figure 4 shows representative snapshots from model simulations at different time points. Simulations start with an APC:naïve T-cell number ratio of 1:10 [35] (with periodic boundary conditions), in particular with three APCs and 30 naïve T-cells. In addition, considering the heterogeneity and the constant change in cell size, we made the simplifying assumption that the APC:naïve T-cell size ratio was of 10:1 [35]. T-cell motility allows for APC-T-cell interactions, crucial for the initiation of an immune response. We can observe that by Day 4 post infection (Day 3 is the initial time point in our simulations) the majority of T-cells established contacts with APC, which enables T-cells to begin their differentiation process. By Day 5 all the APC die, whereas T-cells proliferate extensively (see Supplementary Movie for the whole temporal evolution of the cell population).

Figure 4. Representative simulation snapshots showing the evolution and differentiation of T-cells following the APC encounter. (A) The green arrow shows a naïve T-cell (blue) which turned into a preactivated cell (green) once it came into contact with an APC cell (red); (B) The magenta arrow shows an activated T-cell; and $(\mathbf{C})$ the red arrows show effector T-cells (dark brown). All APCs have died by Day 5. The parameters used for simulations are presented in Tables 3-5, with periodic boundary conditions. See also the Supplementary Movie for the full temporal events (representing 2.5 days of real time; from Day 3 to Day 5.5 post infection). Note that Day 3 is the initial time point in our simulations.

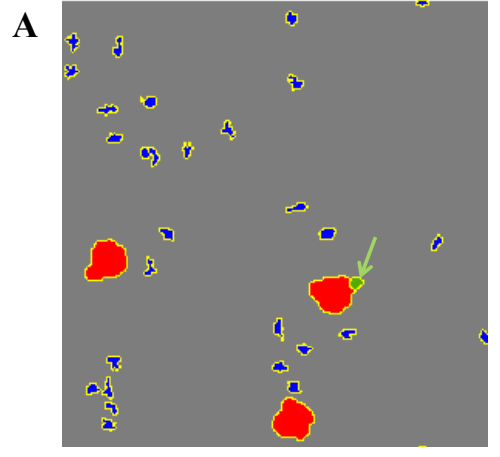

Day 3
B

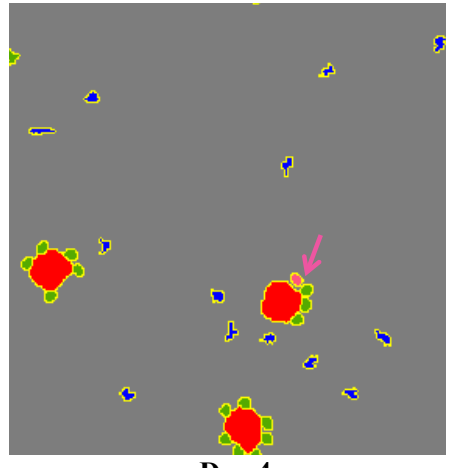

Day 4

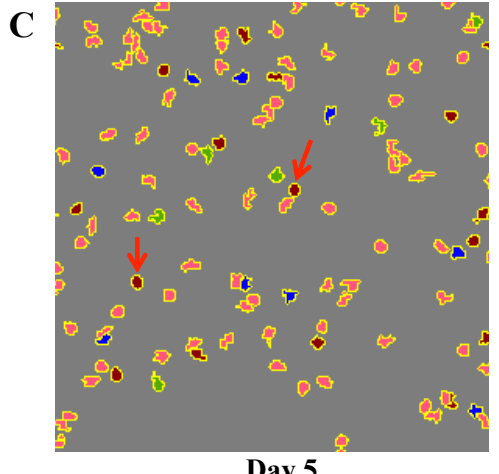

Day 5

Figure 5A shows that our computational results (total T-cell counts) can fit very well in a qualitative manner the results from our experiments (described in Section 2). Note that, the computational cost of the in silico model is the main limiting factor for the two orders of magnitude difference observed between the population sizes in the model and the cell counts in Figure 1. This emerged from both the resolution and coupling of the models (e.g., the ODE model at the subcellular level) and equations (e.g., PDE for IL2 secretion and diffusion) at the different scales. Figure 5B presents computational results for the total cell counts, as well as the counts for each type of T-cell and APC. In particular, it is 
predicted that the number of naive T-cells (blue curve) starts to decrease early enough because of APC encountering and their transition into preactivated cells (green curve). The first activated T-cells start to appear within $\sim 17 \mathrm{~h}$ and proliferate intensively for approximately $30 \mathrm{~h}$. A portion of activated T-cells convert into effector T-cells, which appear after 1.5 days and then strongly proliferate. Effector T-cells outnumber activated cells during the last $\sim 6 \mathrm{~h}$, due to a high differentiation of activated cells and a strong proliferation of effector cells. Therefore, our model can be used to make predictions on the evolution for each of the T- cell stages.

Figure 5. Results at the population level: cell counts. (A) Simulation results in green (right $y$-axis), with the average (of 10 simulations) marked with circles, and experimental results in blue (left $y$-axis), for the total CD8 T-cell counts. Error bars show the SEM; (B) Simulation results present the average (of 10 simulations) and SEM with bars for the total and each CD8 T-cell differentiation stage, as well as APC cell counts. The parameters used for simulations are presented in Tables 3-5. N, Naïve; PA, preactivated; A, activated; E, effector; $T$, total $(N+P A+A+E)$.
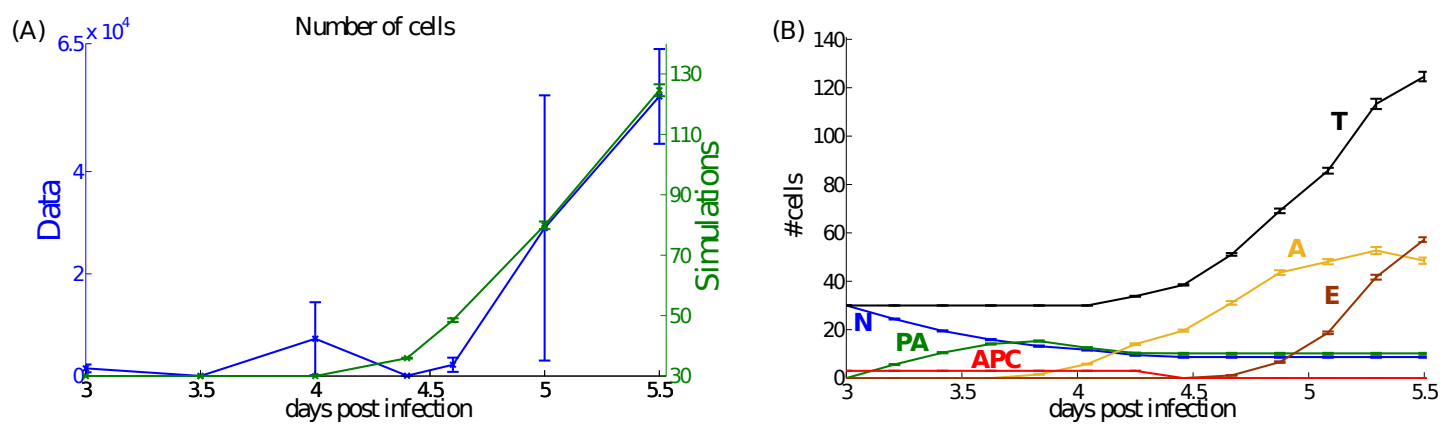

\subsection{Dynamics at the Single Cell Level: Single APC and T Cell Interaction}

In this section, we describe the dynamics at the molecular level, by focusing on the activation phase (TCR activation); that is, when a single APC and a single T-cell come into contact, so that $f(A P C)=1$ (as in Equation (1)), which implies the initiation of the T-cell differentiation process.

First, we make the assumption that IL2 secretion depends only on the TCR signaling (without considering the later effects from activated IL2R, IL2R*). This situation is an approximation of the early times of the activation, when only the APC modifies the state of the molecular regulatory network. That is, IL2 is secreted only when $f(A P C)=1$, and therefore, Equation (9) becomes:

$$
\frac{\partial[I L 2]}{\partial t}=D \nabla^{2}[I L 2]+\lambda_{1} f(A P C)-\delta[I L 2]
$$

To evaluate $\lambda_{1}$ (the secretion rate), its value has been derived such that the $I L 2^{c m}$ (the IL2 at the cell membrane) is $\sim 20 \mathrm{pM}[18]$ once a preactivated T-cell just turns into sn activated T-cell.

Figure 6A,B present the molecular profiles of IL2R*, Tbet, and caspase, along with IL2 expression and secretion, from preactivated and activated T-cells. At $t=0 \mathrm{~h}$ an APC came into contact with a single naïve T-cell which turned into a preactivated T-cell (because of TCR activation). Note that a single preactivated T-cell remains in contact with an APC $(f(A P C)=1)$, however, once it becomes activated it loses contact $(f(A P C)=0)[2,37]$. The preactivated T-cell evolves to its activated phenotype 
once its IL2R* exceeds a threshold (see Table 5). This threshold has been evaluated by the end of a 20-h preactivated T-cell-APC contact having an approximately seven-fold increase from its value at $t=0$ [49]. The IL2R* eventually goes to zero, because the production term in Equation (2) vanishes in contrast to Tbet which keeps on increasing, even though the increase is slowed down following contact loss. Caspase, on the other hand, has slower kinetics and it keeps increasing after the loss of contact, since $f(A P C)$ becomes zero. The activated T-cell may evolve to its effector phenotype once its Tbet concentration exceeds a threshold, which was evaluated such that Tbet reaches an approximately 40-fold increase in about $28 \mathrm{~h}$ [50]. Preactivated T-cells secrete IL2 (Figure 6B; since $f(A P C)=1$ ), and its

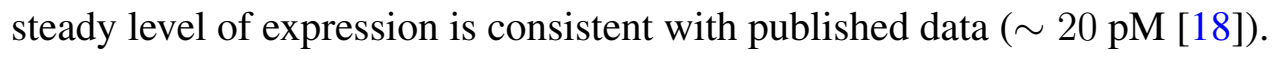

Figure 6. Molecular values of activated IL2R (IL2R*), Tbet and caspase, along with IL2 expression and secretion, from a single preactivated and activated T-cell. At $t=0$, a naïve T-cell comes into contact with an APC. (A) The former differentiates and maintains its preactivated phenotype for $\sim 20 \mathrm{~h}$ (where IL2R* increases $\sim 7$-folds [49]) which further evolves into an activated T-cell. The activated T-cell retains its phenotype for $\sim 28 \mathrm{~h}$ (where Tbet increases $\sim 40$-folds [50]), and eventually turns into an effector phenotype; (B) Preactivated T-cells secrete IL2 at a constant rate (see Equation (10)), and IL2 levels off at $\sim 20 \mathrm{pM}$ [18], whereas activated T-cells stop secreting IL2 due to the loss of contact with APC $(f(A P C)=0$; see Equation (10)). The parameters used for simulations are presented in Tables 3-5.
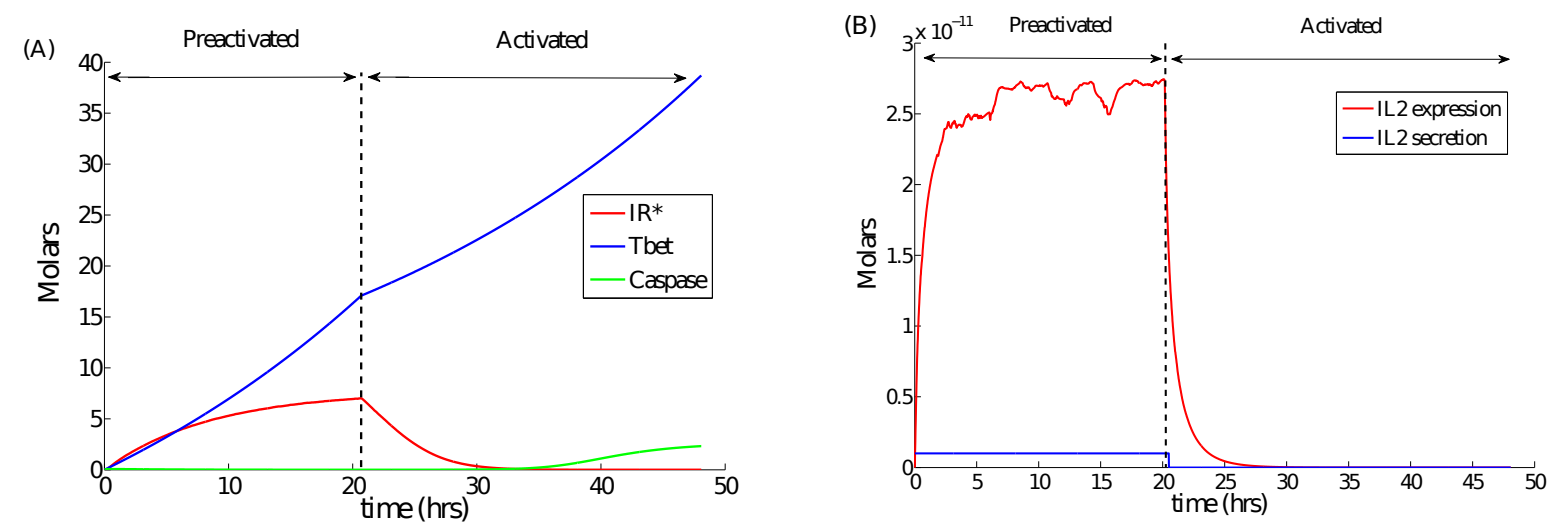

\subsection{A Preliminary Assessment of the Sensitivity of Parameter Values}

We managed to determine most of the numerous parameters of the model (see Tables 4 and 5 , and Section 6.1.2) from the literature and also by making reasonable biological assumptions. Nevertheless, we still rely upon the value of some free parameters $\left(\lambda_{T 3}, \lambda_{c 1}, \lambda_{c 2}, \lambda_{c 3}, \lambda_{c 4}\right)$, which could not be derived from the literature. We therefore examined the impact of some of those free parameters, $\lambda_{c 1}$ and $\lambda_{T 3}$, on the simulation outcomes. These parameters characterize caspase production and Tbet maintenance, respectively. They consequently act directly on T-cell death and differentiation (into effector cells) rates and can then be considered as essential for the CD8 T-cell response.

Figure 7 shows results by varying $\lambda_{c 1}$ (the activation rate of caspase without the role of Fas-FasL) and $\lambda_{T 3}$ (the sensitivity of Tbet to its self-regulation). It is shown that by increasing $\lambda_{c 1}$ (e.g., from 0.01 to 0.02 ), there is increased preactivated, activated and effector cell death. Consequently, in Figure 7A, 
one does not observe the progression of the effector subpopulation by Day 4.5, contrary to what can be observed in Figure 5B, and the overall T-cell production decreases dramatically due to increased cell death. By increasing $\lambda_{T 3}$ (e.g., from 0.01 to 1 ) the activated T-cell population increases since the amount of Tbet remains below the predefined threshold and in such case $\mathrm{T}$ cells are not able to differentiate into effector cells, on the considered time scale (see Figure 7B). The Tbet threshold would be reached later, and so would start the effector T-cell production. One must however note that naïve cell dynamics are not affected by modifications of $\lambda_{c 1}$ and $\lambda_{T 3}$, because these parameters influence biological processes related to proliferating CD8 $\mathrm{T}$ cells.

Figure 7. Results at the population levels: cell counts by varying $\lambda_{c 1}$ and $\lambda_{T 3}$. (A) Increasing $\lambda_{c 1}$ : from 0.01 to 0.02 leads to increased preactivated, activated and effector cell death;(B) Increasing $\lambda_{T 3}$ : from 0.01 to 1 leads to increased proliferation of the activated T-cells with no evolution of effector cells. The results present the average and bars the SEM of 10 simulations. N, Naïve; PA, preactivated; A, activated; E, effector; T, total (N + PA + A $+\mathrm{E})$.
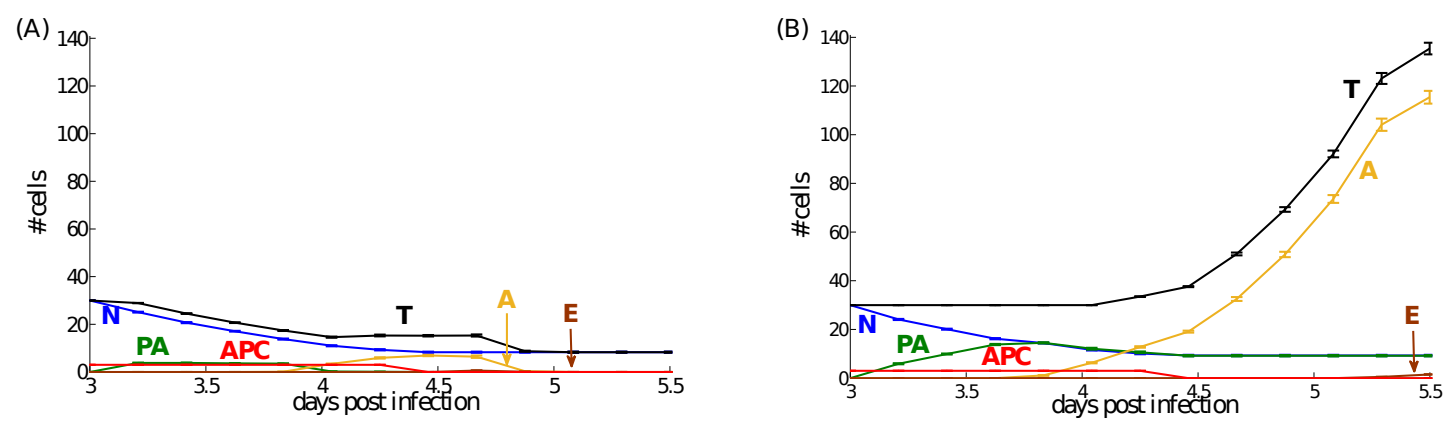

\section{Conclusions}

CD8 T-cells play a major role in protective immunity against many infectious pathogens. The path from naïve precursor to activated and effector CD8 T-cell development begins with interactions with antigen presenting cells (APC).

In this paper, we have developed a 2D multiscale computational model with the aim to understand the dynamics between APC and CD8 T cell interactions in the lymph node (LN), which can lead to the activation, differentiation and clonal expansion of CD8 T-cells. A novel molecular signaling pathway of CD8 T-cells has been presented. Although this is a simplified molecular network, that does not describe the full picture, it does provide the potential for CD8 T-cells to make decisions (differentiate, die by apoptosis) based on their molecular profile. In particular, a CD8 T-cell can convert into its activated phenotype once its activated IL2 receptor level exceeds a threshold, and it can further evolve to its effector phenotype once its Tbet level exceeds a second threshold. Both thresholds were derived based on the time needed for each phenotype to be generated from its precursor, e.g., a preactivated CD8 T-cell can convert into its activated phenotype after a 20-h contact with an APC, and an activated CD8 T-cell needs approximately $28 \mathrm{~h}$ to evolve into its effector phenotype. 
Our integrated model successfully reproduces, qualitatively, the experimental data (total T-cell counts) after deriving most of the parameters (as shown in Tables 4 and 5) and by making biologically reasonable assumptions of the values of the remaining free parameters $\left(\lambda_{T 3}, \lambda_{c 1}, \lambda_{c 2}, \lambda_{c 3}, \lambda_{c 4}\right)$.

This results in giving to our model the capacity of making predictions on the evolution of each CD8 T cell differentiation stage which can be tested experimentally (ongoing experimental work).

Preliminary results on the sensitivity of some of those free parameters have shown that either increasing the activation rate of caspase (without the contribution of Fas-FaL) or the sensitivity of Tbet to its self-regulation results in decreased or increased total cell counts, respectively. Further exploration of the parameter space will be the main focus of our next study in order to investigate different possible outcomes, as well as the sensitivity of the current results to the variation of the fixed parameters.

The induction of CD8 T-cell activation depends on several factors, including cell density, cell motility, the CD8 T-cell:APC ratio in terms of numbers and size, the strength of cell-cell adhesion, IL2 gradients, etc. In the current study, those factors have been carefully taken into account; however, a thorough investigation on the sensitivity of our results based on those factors could reveal noteworthy observations and possible directions for guided experimental investigations.

Our model also considered the role of extracellular IL2 acting on the transition from non-activated to activated IL2R, where the concentration of the latter controls the activation of CD8 T-cells. The simulation results were generated by assuming first that IL2 secretion is mainly dependent on TCR signaling (using Equation (10)). In a subsequent study, we will investigate the effect and sufficiency of both positive and negative feedbacks on IL2 and assess any possible impacts on the population level (evolution and differentiation of CD8 T-cells).

There is a variety of individual-based model approaches (e.g., cellular automata, Cellular Potts Models, hybrid discrete/continuous models). In our study, we used the CompuCell3D open-source simulation environment. Other immune-based frameworks exist (e.g., C-ImmSim [51], Simmune [52]) and could well be applied. It would be very interesting in the future to compare model results emerging from different model frameworks.

Considering the ability of the model to couple the molecular, cellular, and extracellular levels, interesting questions can be addressed regarding how and how much molecular inputs and the microenvironment can affect the behavior at the population level. We believe that our study could pave the way for future studies to integrate single-cell approaches of the fate specification of CD8 T-cells.

\section{Supplementary Information}

\subsection{Parameter Values}

The default parameter values used for our simulations are summarized in Tables 3-5, unless otherwise stated. Below we provide a discussion on how some of those were estimated, and the rest are derived in Section 6.1.2. 


\subsubsection{Cell-Cell Adhesion: Contact Energies $(J)$}

Interactions between neighboring pixels in the CPM have an effective energy, $J$ (as it appears in Equation (7)), which characterizes the strength of cell-cell adhesion (see Table 3). A larger $J$ means more energy is associated with the interface between two cells, which is less energetically favorable, corresponding to weaker adhesivity. Note that in Table 3, when we are referring to a T-cell, it applies to all stages (naïve, preactivated, activated, and effector). It can be observed that $J_{A P C, A}$ and $J_{A P C, E}$ are higher than $J_{A P C, P A}$. We make the assumption that preactivated T cells stay in contact with APCs until they get activated where they lose contact and continue to (randomly) migrate in the domain to search for APCs.

\subsubsection{Parameter Derivation}

For the derived parameters in Tables 4 and 5, we made the following assumptions based on existing literature:

- $\lambda_{1}$ : chosen to be $10^{-12} \mathrm{M} \mathrm{min}^{-1}$, so that $\sim 20 \mathrm{~h}$ [18] are needed for a preactivated cell to become activated, $I L 2^{\mathrm{cm}} \sim 20 \mathrm{pM}$.

- $\lambda_{R 1}$ : it affects the activated IL2R threshold for T-cell activation (from preactivated to activated phenotype), which takes $\sim 20 \mathrm{~h}$ to reach and increases approximately 7-fold [49].

- $\lambda_{R 2}$ : it is a rate chosen to be within the same order as rates $\lambda_{T 2}$ and $\lambda_{c 4}$.

- $\lambda_{T 1}$ : it affects the Tbet threshold for effector T-cells, which takes $\sim 48 \mathrm{~h}$ to reach and increases approximately 40-fold [50].

- $\mu_{I L 2}^{-}$: we assume that this is equal to or at least of the same order as $\mu_{F}^{-}$[19].

- $\mu_{F}^{+}$and $\mu_{F}^{-}$: the equilibrium dissociation rate $\left(K_{d}\right)$ for Fas-FasL is $K_{d}=7 \times 10^{-8} \mathrm{M}$ [53], and for IL2 is $K_{d}=10^{-11} \mathrm{M}$ [48]. Note that $K_{d}=\mu_{F}^{-} / \mu_{F}^{+}$, which implies a weaker interaction of Fas-FasL compared to IL2. By assuming that $\mu_{F}^{-}$of Fas-FasL is approximately of the same order as for IL2, then $\mu_{F}^{-} \sim 10^{-4} \mathrm{~s}^{-1}=6 \times 10^{-3} \mathrm{~min}^{-1}$; and therefore, $\mu_{F}^{+}=0.86 \times 10^{5} \mathrm{M}^{-1} \mathrm{~min}^{-1}$.

- Caspase threshold: we evaluated the caspase threshold to be the value at which the lifespan of the effector T cells is $\sim 60 \mathrm{~h}$ (Supplementary Information of [38]).

For the free parameters, to start with we chose the following values based on the following assumptions:

- $\lambda_{c 1}$ : both $\lambda_{c 1}$ and $\lambda_{T 1}$ represent production rates, and therefore, we choose $\lambda_{c 1}=\lambda_{T 1}$.

- $\lambda_{c 2}$ : due to the lack of any reference values, we assume $\lambda_{c 2} \sim 1 / \lambda_{T 3}$ in order to be dimensionally consistent: $\left[\lambda_{c 2}\right]=M^{-1}$.

- $\lambda_{c 4}$ : due to the lack of any reference values, we assume $\lambda_{c 4} \sim \lambda_{T 2}$ in order to be dimensionally consistent: $\left[\lambda_{c 4}\right]=\min ^{-1}$.

- $\lambda_{T 3}$ : an important biological question based on which we tried to find a reasonable value to start with for $\lambda_{T 3}$, is whether and when an activated T cell can evolve to an effector phenotype (with positive and non-zero Tbet). From Equation (3), we can get an upper bound for $\lambda_{T 3}$; that is, $\lambda_{T 3}<\frac{T b\left(\lambda_{T 2}-k_{T}\right)}{k_{T}}$. By using the value of Tbet at the time when a preactivated $\mathrm{T}$ cell becomes activated, that gives $\lambda_{T 3}<1.24$ (an upper bound for $\lambda_{T 3}$ ). 


\section{Acknowledgments}

The authors would like to thank Camille Pommier for her help in designing an early version of a multiscale model, Alain Trautman for his help in designing early versions of the molecular network, Xuefeng Gao for his valuable comments on the manuscript, and all members of the ANR funded PrediVac Project for helpful discussions throughout the project. The authors thank the Centre de Calcul de l'Institut National de Physique Nucléaire et de Physique des Particules de Lyon (CC-IN2P3), for granting us access to their computing resources, and especially Pascal Calvat, for his invaluable help with installing CompuCell3D on their machines. Special thanks also go to Maciej Swat for his assistance in the CompuCell3D installation on the CC-IN2P3 machines and all of the CompuCell3D group members for their continuous scientific support. This work has been supported by ANR grant PrediVac ANR-12-RPIB-0011, the Finovi Fondation, the Rhône-Alpes Complex Systems Institute (IXXI), the Université Claude Bernard Lyon 1, the Fonds Europeen de Developpement Regional, and institutional grants from the Institut National de la Sante Et de la Recherche Medicale.

\section{Author Contributions}

Sotiris A.Prokopiou performed the model construction and parameter estimation as well as parameter value analysis, together with Olivier Gandrillon, Samuel Bernard, Loic Barbarroux and Fabien Crauste. Jacqueline Marvel, Christophe Arpin and Julien Mafille participated in the acquisition of the in vivo data. Yann Leverrier, Olivier Gandrillon and Christophe Arpin participated in the parameter estimation process. Olivier Gandrillon and Fabien Crauste conceived the study and participated in its design and coordination. Sotiris A.Prokopiou, Olivier Gandrillon and Fabien Crauste wrote the manuscript, with the help of Jacqueline Marvel and Christophe Arpin. All authors read and approved the final manuscript.

\section{Conflicts of Interest}

The authors declare no conflicts of interest.

\section{References}

1. Matheu, M.; Teijaro, J.; Walsh, K.; Greenberg, M.; Marsolais, D.; Parker, I.; Rosen, H.; Oldstone, M.; Cahalan, M. Three phases of CD8 T cell response in the lung following H1N1 influenza infection and sphingosine 1 phosphate agonist therapy. PLoS One 2013, 8, e58033. doi:10.1371/journal.pone.0058033.

2. Bousso, P. T-cell activation by dendritic cells in the lymph node: Lessons from the movies. Nat. Rev. Immunol. 2008, 8, 675-684.

3. Cui, W.; Kaech, S. Generation of effector CD8+ T cells and their conversion to memory T cells. Immunol. Rev. 2010, 236, 151-166.

4. Williams, M.; Bevan, M. Effector and memory CTL differentiation. Annu. Rev. Immunol. 2007, 25, 171-192. 
5. Li, Q.; Skinner, P.; Ha, S.; Duan, L.; Mattila, T.; Hage, A.; White, C.; Barber, D.; O’Mara, L.; Southern, P.; et al. Visualizing antigen-specific and infected cells in situ predicts outcomes in early viral infection. Science 2009, 323, 1726-1729.

6. Mueller, S.; Germain, R. Stromal cell contributions to the homeostasis and functionality of the immune system. Nat. Rev. Immunol. 2009, 9, 618-629.

7. Bajenoff, M.; Egen, J.; Koo, L.; Laugier, J.; Brau, F.; Glaichenhaus, N.; Germain, R. Stromal Cell Networks Regulate Lymphocyte Entry, Migration, and Territoriality in Lymph Nodes. Immunity 2006, 25, 989-1001.

8. Buchholz, V.; Graf, P.; Busch, D. The smallest unit: effector and memory CD8(+) T cell differentiation on the single cell level. Front Immunol. 2013, 4, doi:10.3389/fimmu.2013.00031.

9. Antia, R.; Bergstrom, C.; Pilyugin, S.; Kaech, S.; Ahmed, R. Models of CD8+ responses: 1. What is the antigen-independent proliferation program. J. Theor. Biol. 2003, 221, 585-598.

10. Terry, E.; Marvel, J.; Arpin, C.; Gandrillon, O.; Crauste, F. Mathematical model of the primary CD8 T cell immune response: stability analysis of a nonlinear age-structured system. J. Math. Biol. 2012, 65, 263-291.

11. Beltman, J.; Maree, A.; de Boer, R. Spatial modelling of brief and long interactions between $\mathrm{T}$ cells and dendritic cells. Immunol. Cell Biol. 2007, 85, 306-314.

12. Glazier, J.; Graner, F. Simulation of the differential adhesion driven rearrangement of biological cells. Phys. Rev. E 1993, 47, 2128-2154.

13. Gong, C.; Mattila, J.; Miller, M.; Flynn, J.; Linderman, J.; Kirschner, D. Predicting lymph node output efficiency using systems biology. J. Theor. Biol. 2013, 335, 169-184.

14. Riggs, T.; Walts, A.; Perry, N.; Bickle, L.; Lynch, J.; Myers, A.; Flynn, J.; Linderman, J.; Miller, M.; Kirschner, D. A comparison of random vs. chemotaxis driven contacts of $\mathrm{T}$ cells with dendritic cells during repertoire scanning. J. Theor. Biol. 2008, 250, 732-751.

15. Webster, B.; Ekland, E.; Agle, L.; Chyou, S.; Ruggieri, R.; Lu, T. Regulation of lymph node vascular growth by dendritic cells. J. Exp. Med. 2006, 203, 1903-1913.

16. Bogle, G.; Dunbar, P. Agent-based simulation of T-cell activation and proliferation within a lymph node. Immunol. Cell Biol. 2010, 88, 172-179.

17. Baldazzi, V.; Paci, P.; Bernaschi, M.; Castiglione, F. Modeling lymphocytes homing and encounters in lymph nodes. BMC Bioinforma. 2009, 10, doi:10.1186/1471-2105-10-387.

18. Hofer, T.; Krichevsky, O.; Altan-Bonnet, G. Competition for IL-2 between Regulatory and Effector $\mathrm{T}$ Cells to Chisel Immune Responses. Front Immunol. 2012, 3, 268. doi:10.3389/fimmu.2012.00268.

19. Feinerman, O.; Jentsch, G.; Tkach, K.; Coward, J.; Hathorn, M.; Sneddon, M.; Emonet, T.; Smith, K.; Altan-Bonnet, G. Single-cell quantification of IL-2 response by effector and regulatory $\mathrm{T}$ cells reveals critical plasticity in immune response. Mol. Syst. Biol. 2010 , 6, doi:10.1038/msb.2010.90.

20. Hoyer, K.; Dooms, H.; Barron, L.; Abbas, A. Interleukin-2 in the development and control of inflammatory disease. Immunol. Rev. 2008, 226, 19-28. 
21. McLane, L.; Banerjee, P.; Cosma, G.; Makedonas, G.; Wherry, E.; Orange, J.; Betts, M. Differential localization of $\mathrm{T}$-bet and Eomes in CD8 $\mathrm{T}$ cell memory populations. J. Immunol. 2013, 7, 3207-3215.

22. Martins, G.; Calame, K. Regulation and functions of Blimp-1 in T and B lymphocytes. Annu. Rev. Immunol. 2008, 26, 133-169.

23. Yeo, C.; Fearon, D. T-bet-mediated differentiation of the activated CD8+ T cell. Eur. J. Immunol. 2011, 41, 60-66.

24. Szabo, S.; Kim, S.; Costa, G.; Zhang, X.; Fathman, C.; Glimcher, L. A novel transcription factor, T-bet, directs Th1 lineage commitment. Cell 2000, 100, 655-669.

25. Hwang, E.; Hong, J.; Glimcher, L. IL-2 production in developing Th1 cells is regulated by heterodimerization of RelA and T-bet and requires T-bet serine residue 508. J. Exp. Med. 2005, 202, 1289-1300.

26. Ewings, K.; Wiggins, C.; Cook, S. Bim and the pro-survival Bcl-2 proteins: Opposites attract, ERK repels. Cell Cycle 2007, 6, 2236-2240.

27. Kelly, J.; Spolski, R.; Imada, K.; Bollenbacher, J.; Lee, S.; Leonard, W. A role for Stat5 in CD8+ T cell homeostasis. J. Immunol. 2003, 170, 210-217.

28. Sullivan, B.; Juedes, A.; Szabo, S.; von Herrath, M.; Glimcher, L. Antigen-driven effector CD8 T cell function regulated by T-bet. Proc. Natl. Acad. Sci. USA 2003, 100, 15818-15823.

29. Bouillet, P.; O'Reilly, L. CD95, BIM and T cell homeostasis. Nat. Rev. Immunol. 2009, 9, 514-519.

30. Kanhere, A.; Hertweck, A.; Bhatia, U.; Gökmen, M.; Perucha, E.; Jackson, I.; Lord, G.; Jenner, R. T-bet and GATA3 orchestrate Th1 and Th2 differentiation through lineage-specific targeting of distal regulatory elements. Nat. Commun. 2012, 3, doi:10.1038/ncomms2260.

31. Shin, H.; Lee, J.; Park, S.; Chang, J.; Lee, C.W. T-bet expression is regulated by EGR1-mediated signaling in activated T cells. Clin. Immunol. 2009, 131, 385-394.

32. Andersen, M.; Schrama, D.; Thor Straten, P.; Becker, J. Cytotoxic T cells. J. Invest. Dermatol. 2006, 126, 32-41.

33. Liao, W.; Lin, J.; Leonard, W. Interleukin-2 at the crossroads of effector responses, tolerance, and immunotherapy. Immunity 2013, 38, 13-25.

34. Swat, M.; Thomas, G.; Belmonte, J.; Shirinifard, A.; Hmeljak, D.; Glazier, J. Multi-Scale Modeling of Tissues Using CompuCell3D. Comput. Methods Cell Biol. Methods Cell Biol. 2012, $110,325-366$.

35. Miller, M.; Hejazi, A.; Wei, S.; Cahalan, M.; Parker, I. T cell repertoire scanning is promoted by dynamic dendritic cell behavior and random $\mathrm{T}$ cell motility in the lymph node. Proc. Natl. Acad. Sci. USA 2004, 101, 998-1003.

36. Bauer, A.; Jackson, T.; Jiang, Y. A cell-based model exhibiting branching and anastomosis during tumor-induced angiogenesis. Biophys. J. 2007, 92, 3105-3121.

37. Bousso, P.; Robey, E. Dynamics of CD8+ T cell priming by dendritic cells in intact lymph nodes. Nat. Immunol. 2003, 4, 579-585. 
38. Linderman, J.; Riggs, T.; Pande, M.; Miller, M.; Marino, S.; Kirschner, D. Characterizing the dynamics of $\mathrm{CD} 4+\mathrm{T}$ cell priming within a lymph node. J. Immunol. 2010, 184, doi:10.4049/jimmunol.0903117.

39. $\mathrm{Wu}, \mathrm{J} . ;$ Tzanakakis, E. Contribution of stochastic partitioning at human embryonic stem cell division to NANOG heterogeneity. PLoS One 2012, 7, e50715. doi:10.1371/journal.pone.0050715.

40. Buchholz, V.; Flossdorf, M.; Hensel, I.; Kretschmer, L.; Weissbrich, B.; Graf, P.; Verschoor, A.; Schiemann, M.; Hofer, T.; Busch, D. Disparate individual fates compose robust CD8+ T cell immunity. Science 2013, 340, 630-635.

41. Gerlach, C.; Rohr, J.; Perie, L.; van Rooij, N.; van Heijst, J.; Velds, A.; Urbanus, J.; Naik, S.; Jacobs, H.; Beltman, J.; et al. Heterogeneous differentiation patterns of individual CD8+ T cells. Science 2013, 340, 635-639.

42. Huh, D.; Paulsson, J. Non-genetic heterogeneity from stochastic partitioning at cell division. Nat. Genet. 2011, 43, 95-100.

43. Lanzavecchia, A.; Sallusto, F. Lead and follow: The dance of the dendritic cell and T cell. Nat. Immunol. 2004, 5, 1201-1202.

44. Lindquist, R.; Shakhar, G.; Dudziak, D.; Wardemann, H.; Eisenreich, T.; Dustin, M.; Nussenzweig, M. Visualizing dendritic cell networks in vivo. Nat. Immunol. 2004, 5, 1243-1250.

45. Sayar, D.; Ketzinel, M.; Gerez, L.; Silberberg, C.; Reshef, A.; Kaempfer, R. Expression of the human IL-2 receptor on lymphocytes involves rapid turnover of its p55 alpha-subunit. J. Immunol. 1990, 145, 2946-2949.

46. Yates, A.; Callard, R.; Stark, J. Combining cytokine signalling with T-bet and GATA-3 regulation in Th1 and Th2 differentiation: a model for cellular decision-making. J. Theor. Biol. 2004, 231, 181-196.

47. Larsson, E.; Sander, C.; Marks, D. mRNA turnover rate limits siRNA and microRNA efficacy. Mol. Syst. Biol. 2010, 6, doi:10.1038/msb.2010.89.

48. Smith, K. The structure of IL2 bound to the three chains of the IL2 receptor and how signaling occurs. Med. Immunol. 2006, 5, doi:10.1186/1476-9433-5-3.

49. Mempel, T.; Henrickson, S.; von Andrian, U. T cell priming by dendritic cells in lymph nodes occurs in three distinct phases. Nature 2004, 427, 154-159.

50. Cruz-Guilloty, F.; Pipkin, M.; Djuretic, I.; Levanon, D.; Lotem, J.; Lichtenheld, M.; Groner, Y.; Rao, A. Runx3 and T-box proteins cooperate to establish the transcriptional program of effector CTLs. J. Exp. Med. 2009, 206, 51-59.

51. Rapin, N.; Lund, O.; Bernaschi, M.; Castiglione, F. Computational Immunology Meets Bioinformatics: The Use of Prediction Tools for Molecular Binding in the Simulation of the Immune System. PLoS One 2010, 5, e9862. doi:10.1371/journal.pone.0009862. 
52. Angermann, B.; Klauschen, F.; Garcia, A.; Prustel, T.; Zhang, F.; Germain, R.; Meier-Schellersheim, M. Computational modeling of cellular signaling processes embedded into dynamic spatial contexts. Nat. Meth. 2012, 9, 283-289.

53. Starling, G.; Bajorath, J.; Emswiler, J.; Ledbetter, J.; Aruffo, A.; Kiener, P. Identification of amino acid residues important for ligand binding to Fas. J. Exp. Med. 1997, 185, 1487-1492.

(c) 2014 by the authors; licensee MDPI, Basel, Switzerland. This article is an open access article distributed under the terms and conditions of the Creative Commons Attribution license (http://creativecommons.org/licenses/by/4.0/). 